\title{
Cost-Effectiveness of Treatment Strategies for Primary Operable Pancreatic Head Adenocarcinoma: Do We Have More Scientific Evidence to Call for Further Centralization of Care?
}

\author{
Csaba Gajdos, MD and Richard Schulick, MD, MBA \\ Section of GI, Tumor and Endocrine Surgery, Department of Surgery, University of Colorado Anschutz Medical Campus, \\ Aurora, $\mathrm{CO}$
}

According to recent statistics from the American Cancer Society, the number of newly diagnosed pancreas cancer cases was 45,000 in 2010. The annual number of patients succumbing to this disease continues to nearly equal the number of newly diagnosed cases. Despite a number of improvements in the diagnosis and treatment of these patients, overall survival from pancreas cancer remains poor. Whereas certain centers of excellence have reported 5 -year survival rates up to $36 \%$ in highly selected patients in the setting of curative intent resection, survival curves using data from large population-based datasets reflect a substantially worse outcome. ${ }^{1,2}$

Currently accepted treatment modalities for pancreas cancer include surgery, chemotherapy, radiation, or palliative care/no treatment. The only potential chance for cure or long-term survival for patients with pancreatic malignancies is a curative intent resection. ${ }^{3}$ The majority of patients with newly diagnosed operable disease will require a Whipple procedure. Although the safety of these operations has come a long way in the past few decades, pancreaticoduodenectomies are still complex operations with a chance for perioperative morbidity and mortality. ${ }^{4}$ Adverse postoperative events are known to increase medical costs substantially.

Some papers in the literature have reported survival of nonsurgically treated patients diagnosed with pancreas cancer. Chemotherapy with radiation can result in survival rates in the 12 months range in the nonmetastatic setting upon presentation. ${ }^{5}$ This overall survival is only a few

(C) Society of Surgical Oncology 2012

Published Online: 5 October 2012

R. Schulick, MD, MBA

e-mail: richard.schulick@ucdenver.edu months shorter than survival reported from major US centers following curative intent surgery. ${ }^{6,7}$ With rising health care cost in our country and increasing scrutiny from insurance companies of how health care is delivered, examining the cost-benefit ratio of various treatment modalities implemented in pancreas cancer treatment could not be timelier.

In this issue of the Annals of Surgical Oncology, Abbott et al. ${ }^{8}$ compare the cost-effectiveness of various treatment strategies for primary operable pancreatic head adenocarcinoma. To our knowledge, this is the first study to compare costs and outcomes associated with various treatment strategies for pancreas cancer. Their decision model compared six strategies: no treatment, radiation therapy only, chemotherapy only, chemotherapy plus radiation, surgery alone, and surgery plus adjuvant therapy. Outcomes and probabilities were identified by using the National Cancer Data Base and the ACS National Surgical Quality Improvement Program, in addition to the literature. Costs were estimated using Medicare payments. Low- and highperforming hospitals were selected using the following variables: perioperative mortality, overall complications, "unresectable at operation," margin positivity rate, and median survival.

Although the paper has the usual shortcomings of working with large datasets, the authors have to be commended for their efforts. Not surprisingly, surgery plus adjuvant therapy, chemotherapy alone, or no treatment were the only viable strategies with regards to cost-effectiveness. The elevated cost of surgery plus adjuvant therapy was largely due to poor outcomes associated with this best treatment available. While the cost of postsurgical complications was considered, the cost of complications related to nonsurgical treatment modalities was not measured. 
Most importantly, increased survival of patients undergoing curative intent operations had the greatest impact on the cost-effectiveness of surgical intervention. Treatment in low-performing centers was more expensive compared with treatment in high-performing centers.

We agree with the authors conclusions that although surgery plus adjuvant therapy is costly, this treatment combination could result in long-term survival or potential cure, especially if postoperative adverse events are minimized. We also agree that our society should have willingness to pay threshold for potentially curative interventions compared with other treatment modalities that have never been shown to result in long-term survivors. Based on results from this paper, further improvement in cost-effectiveness with the following strategies should be implemented and routinely monitored in every hospital performing Whipple operations for pancreas cancer (percentages in parenthesis are data from the paper that we considered too high or too low):

1) Consider decreasing the rate of preoperative biliary stenting, because there is high level of scientific evidence for this intervention to increase postoperative complications significantly $(67 \%) .{ }^{9}$ Although this might not be feasible in patients presenting with obstructive jaundice in the rural/small-town setting, because surgical care is not immediately available, high-volume centers should have the capacity to perform surgery in a more timely fashion.

2) Improve patient selection for curative intent operation via high-quality preoperative workup, including dedicated pancreas protocol cat scan read by well-trained radiologists and surgeons, to decrease nontherapeutic laparotomies (unresectable at operation: $17 \%$ ).

3) Increase truly margin-negative resection rate (84\% in this paper, which in real life is likely lower because careful examination of the retroperitoneal resection margin is still not standard in many hospitals) via proper surgical technique, careful skeletonization of superior mesenteric artery, appropriate orientation of the surgical specimen, including the retroperitoneal margin, and liberal use of intraoperative frozen sections on pancreas and bile duct margins interpreted by well-trained gastrointestinal pathologists.

4) Decrease major surgical complication rates that significantly increase treatment cost (hospital reimbursement for uncomplicated resection DRG 407: \$23,430; for major complication following resection DRG 405: $\$ 54,460$ ) and likely significantly contribute to delaying or completely omitting adjuvant therapy (41\%) via high-quality surgical and postoperative care in dedicated surgical units with experienced nursing staff. ${ }^{8}$
5) Consider decreasing the rate of adjuvant radiation for early-stage pancreas cancer resected with truly negative surgical margins (see \#3), because the added cost of radiation is substantial with unclear benefits (stage I-IIa: $81 \%$ radiation rate for early-stage marginnegative pancreas cancer and $85 \%$ for marginpositive ones; pancreaticoduodenectomy surgical reimbursement $\$ 3,057$ vs. radiation treatment reimbursement $\$ 8,582) .{ }^{8,10}$

We believe that these are modifiable perioperative variables, and continuous monitoring of the above-mentioned "performance measures" by every hospital is crucial. This would likely lead to improved cost-effectiveness in the multidisciplinary treatment of primary operable pancreas cancer. This optimized care may be more achievable in high-performing centers, resulting in significant savings of diminishing health-care dollars.

\section{REFERENCES}

1. Evans BD, Varadhachary GR, Crane $\mathrm{CH}$, et al. Preoperative gemcitabine-based chemoradiation for patients with resectable adenocarcinoma of the pancreatic head. J Clin Oncol. 2008;26: 3496-502.

2. Mayo SC, Austin DF, Sheppard BC, et al. Adjuvant therapy and survival after resection of pancreatic adenocarcinoma. Cancer. 2010:116:2932-2940.

3. Schnelldorfer T, Ware AL, Sarr MG, et al. Long-term survival after pancreaticoduodenectomy for pancreatic adenocarcinoma. Ann Surg. 2008;247(3):456-462.

4. Cameron JL, Taylor SR, Coleman J, Belcher KA. One thousand consecutive pancreaticoduodenectomies. Ann Surg. 2006;244(1): $10-15$.

5. Varadhachary GR, Wolff RA, Crane $\mathrm{CH}$, et al. Preoperative gemcitabine and cisplatin followed by gemcitabine based chemoradiation for resectable adenocarcinoma of the pancreatic head. J Clin Oncol. 2008;26:3487-3495.

6. Fatima J, Schnelldorfer T, Barton J, et al. Pancreaticoduodenectomy for ductal adenocarcinoma. Arch Surg. 2010;142(2): 167-172.

7. Herman JM, Swartz MJ, Hsu CC, et al. Analysis of fluorouracilbased adjuvant chemotherapy and radiation after pancreaticoduodenectomy for ductal adenocarcinoma of the pancreas: results of a large prospectively collected database at the Johns Hopkins Hospital. J Clin Oncol. 2008;26:3503-3510.

8. Abbott D, Merkow R, Cantor S, et al. Cost-effectiveness of treatment strategies for pancreatic head adenocarcinoma and potential opportunities for improvement. Ann Surg Oncol. 2012. doi:10.1245/s10434-012-2610-1.

9. Van der Gaag NA, Rauws EAJ, van Eijick CHJ, et al. Preoperative biliary drainage for cancer of the head of the pancreas. $N$ Engl J Med. 2010;362:129-137.

10. Neptolemos JP, Stocken DD, Friess H, et al. A randomized trial of chemoradiotherapy and chemotherapy after resection of pancreatic cancer. N Engl J Med. 2004;350:1200-1210. 